\title{
Assessment and appliction ofWhole-herd-Sampling, qPCR-based-Testing, and Precision-Removal method in Eliminating ASFv in Four Large Swine Herds in China
}

\section{Zhichun Jason Yan ( $\nabla$ jasonynh@126.com )}

Swine Research Institute of New Hope Liuhe Co., Ltd

Xiaowen Li

Swine Research Institute of New Hope Liuhe Co., Ltd

Weisheng Wu

Swine Research Institute of New Hope Liuhe Co., Ltd

Peng Li

Swine Research Institute of New Hope Liuhe Co., Ltd Junxian Li

Swine Research Institute of New Hope Liuhe Co., Ltd

Wenchao Gao

Swine Research Institute of New Hope Liuhe Co., Ltd

Jincheng Yu

Swine Research Institute of New Hope Liuhe Co., Ltd

Mingyu Fan

Swine Research Institute of New Hope Liuhe Co., Ltd

\section{Lixiang Kang}

Swine Research Institute of New Hope Liuhe Co., Ltd

Yunzhou Wang

Swine Research Institute of New Hope Liuhe Co., Ltd

\section{Guiqiang Yuan}

Swine Research Institute of New Hope Liuhe Co., Ltd

Qiannan Yu

Swine Research Institute of New Hope Liuhe Co., Ltd Jing Ren

Swine Health Data and Intelligent Monitoring Project Laboratory (Dezhou, China)

\section{Zhong Yan}

Swine Research Institute of New Hope Liuhe Co., Ltd

\section{Liqiang Zhu}

Swine Research Institute of New Hope Liuhe Co., Ltd 


\section{Jintao Li}

Swine Research Institute of New Hope Liuhe Co., Ltd

\section{Xiaoyang Zhang}

Swine Research Institute of New Hope Liuhe Co., Ltd

\section{Zhenwen Shao}

Swine Research Institute of New Hope Liuhe Co., Ltd

\section{Qingyuan Liu}

Swine Research Institute of New Hope Liuhe Co., Ltd

\section{Weiwei Zheng}

Swine Research Institute of New Hope Liuhe Co., Ltd

\section{Shiran Fan}

Swine Research Institute of New Hope Liuhe Co., Ltd

\section{Yakuan Huang}

Swine Research Institute of New Hope Liuhe Co., Ltd

\section{Jing Chen}

Swine Research Institute of New Hope Liuhe Co., Ltd

\section{John Deen}

University of Minnesota, St. Paul, (MN, the United States)

\section{Case Report}

Keywords: African swine fever virus, quantitative PCR, test removal

Posted Date: April 6th, 2022

DOl: https://doi.org/10.21203/rs.3.rs-954531/v2

License: (c) (i) This work is licensed under a Creative Commons Attribution 4.0 International License. Read Full License 


\section{Abstract}

\section{Background}

Since the first report of ASFV in China in 2018, conventional whole herd depopulation method to control ASF has proved unwieldly because of high production intensity and complex trade network. To provide an alternative to conventional methods, we evaluated the feasibility of implementating an extensive sampling method and qPCR tests to determine the status of ASFV in herds, with a rapid response to identified outbreaks.

\section{Case presentations}

By assessing and applying these methods without whole herd depopulation, we successfully controlled ASF and eliminated the virus from 4 large swine herds. The time to negative herd was 19,28, 14, and 1 days in farms 1-4 respectively. Retention rates of pigs of farm 1 to farm 4 was $69.7 \%, 65 \%, 99.4 \%$ and $99.72 \%$ respectively.

\section{Conclusions}

We anticipated that this innovative method would replace the conventional stamping out one and greatly facilitate the control and eradication of ASFV in China and worldwide.

\section{Background}

The first outbreak of ASF in China was reported on 3rd August, $2018^{1}$. Without vaccine, the only available tool to prevent ASFV is the implementation of strict bio-security measures at regional and farm level. The response of the veterinary authorities was consistent with the OIE guidelines ${ }^{2}$. This means that the Ministry of Agriculture and Rural Affairs (MARA) of China required depopulation of infected and proximal farms once ASFV was reported ${ }^{3}$. However, it soon became apparent, that these measures were not effective in China for a number of reasons. These include the high density of pig farms, of which the vast majority were small-scale producers that were connected with each other via highly complex pig and pork trade networks. There may also have been inefficiencies in the response by the industry and government in the early stage of the epidemic. Unlike for other pig diseases such as pseudorabies, there is not any scientific literature that describes the elimination ASFV within a herd without of whole herd depopulation.

Informal reports from affected farmers indicate that the transmission pattern of ASFV in large herds differs from that of other major swine viral diseases. Published information indicates that after infection, time to the manifestation of typical clinical signs varied considerably among pigs, as well as within-pen contact pigs and cross-pen contact pigs. ASFV DNA detection in oral, nasal and rectal swab samples occurred between 0 and 2 days before onset of clinical signs ${ }^{4,5}$, suggesting that early detection of viral DNA by qPCR prior to clinical signs may be possible as part of active within-herd ASF surveillance. 
It was observed that ASFV spreads relatively slowly within a herd following introduction, with estimated within-pen basic reproduction ratio (R0) of approximately 2.8 and between-pen R0 of approximately $1.4^{6}$. Moreover, environmental contamination of ASFV can be relatively light, as was shown that introduction of negative pigs into contaminated pens 3, 5, 7 days after infected pigs were removed did not cause infection in this study ${ }^{5}$. In addition, aerosol transmission of ASFV appears to be unlikely to occur as the half-life of ASFV in air was estimated to be $14 \sim 19$ minutes $^{7}$. Moreover, we observed that most infections within barns were associated with common routes of contact via direct contact, feces or saliva. This published scientific information together with the impression from farms in China indicated that it might be possible to achieve ASFV eradication within a herd by using a test-and-removal method.

Surveillance would be most practical if pooled samples could be used for diagnosis without compromising sensitivity, and one study using pooled EDTA blood to test for ASFV by qPCR indicated that it may be feasible ${ }^{8}$.

Our current approach is based on a combination of published research on ASFV together with our own experience with ASFV management in large pig herds. In this study we assessed the feasibility of method in eliminating ASFV Georgia 2007/1 Strain using detailed results from 1 finishing and 3 sow herds.

\section{Case Presentation}

\section{Validation of maximum number of sample pooling for achieving acceptable sensitivity of ASFV DNA detection}

When the initial CT was approximately 20, even after 2048 times of dilution, the final CT was still around 32 (Figure 1). However, when the primary CT was set at around 33, the final CT values became around 38.5 after 16 folds of dilution. Likewise, as the dilution increased, the variation increased dramatically.

\section{TTNH (Time to negative herd) of a finishing herd and three sow herds}

Time to negative herd(TTNH), which is literally defined as time period from the day of first ASFV detection to the day of last ASFV detection in pigs and environment, in Farm 1 was 19 days (Figure 2A). On farm 1 (a finishing herd), Ct values of ASFV-qPCR in lymph nodes from 4 dead pigs were 20.97 (room 1), 20.39 (room 3), 32.67(room 6), 29.85(room 3), respectively on day 0 (May 14 ${ }^{\text {th }}, 2019$ ) (Figure S4).

Considering the low $\mathrm{Ct}$ values, the whole pen was depopulated in a bio-secure manner on day 0 . On day 2 , samples from three dead pigs and two vehicles transporting pigs were found to be qPCR positive. The whole herd sampling, which yielded 241 pig samples, 135 enviromental samples, and testing on day 4 showed that only the environmental sample from the pig transfer corridor was qPCR positive with a $\mathrm{Ct}$ value of 32.55. After precision removal of pigs and thorough cleaning and disinfection of the pig contact area, the herd remained negative for 14 consecutive days (approximately two incubation periods) until 
Pen 10 in Room 5 was found to be qPCR positive in oral fluid samples in day 19. The method was carried out again and the herd restored its negative status by removing a total of 1282 pigs and remained ASFV negative since day 19 until the batches were marketed.

TTNH for Farm 2 was 28 days (figure 2B). In Production Line 1 of Farm 2, one out of twenty one samples, ASFV was first detected by qPCR (Ct value of 22.7) in a Lymph node sample from a dead pig in Stall G17 of Gestation Room 1 in July 11 of 2019 (day 0). Later in the same day, whole herd sampling and testing of Line 1 were performed. Pooled samples of Row A in Gestation Room 1 and Row K of Gestation Room 2 were found ASFV qPCR positive with a Ct value of 34.71 and 34.87, respectively. We observed an intermittent mode of qPCR positive results. The whole surface environmental samples in Gestation Room 1 was found to be qPCR positive almost every day until day 28 when disinfectant sodium hypochlorite was applied. Pigs in Gestation Room 2 restored negative status from day 6, even though the whole surface of environmental samples were found ASFV qPCR positive in day 8 and day 26 and day 27. Samples on 2 working staff were found to be qPCR positive at day 4 and day 23, respectively. The whole herd of Production Line 1 restored negative status from day 28 and remained negative ever since last detection (data not shown). An intermittent mode of ASFV detection was found probably due to not-fully implemented paired sampling.

TTNH for Farm 3 was 14 days. (figure 2C). In Farm 3, a nasal, oral and rectal(NOR) swab sample from an off-feed sow was found ASFV qPCR positive with Ct values of 35.65 (Stall A-87) in day 0 (January $28^{\text {th }}$, 2020). The Ct value of qPCR results from lymph node sample was 32.39. All samples from clinically abnormal pigs, whole surface of environment, personnel, and supplies were found qPCR negative after precision removal and thorough cleaning and disinfection. One interesting finding was that the feeder outlet from A87 was shown qPCR positive with a Ct value of 39.53 on day 14. Farm 3 remained negative since day 14 after the feeder was decontaminated by sodium hypochlorite.

TTNH for Farm 4 was 1 day. (figure 2D). In farm 4, the nasal, oral and rectal(NOR) swab samples from an off-feed sow was found ASFV qPCR positive with Ct values of 26.12 (Stall H26) on day 0 (June 4th, 2020). The Ct value of qPCR results from lymph node samples was 32.39 (data not shown). After precision removal and thorough cleaning and disinfection, the herd restored its negative status in day 1 and remained negative since then. (data not shown).

\section{Retention rate}

The retention rate (=number of retained sows/ number of sows prior to ASFV detection) of farm 1 to farm 4 was determined to be $69.7 \%, 65 \%, 99.4 \%, 99.72 \%$ respectively. (figure 3 )

\section{Discussions And Conclusions}

Without vaccines, an innovative and practical way to deal with ASFV was needed due to unsuccessful and expensive application of conventional standard culling measures. By combining scientific knowledge 
of ASFV with field attempts, we successfully developed a systematic "Whole-herd Sampling, qPCR-based Testing, and Precision Removal" method that successfully eradicated Georgia 2007/1 strain of ASFV in several swine herds.

ASFV was found to be relatively slow in transmission after infection occurs. One study showed that the within pen RO of ASFV was estimated to be $2.8^{3}$. Our field practices and observations were consistent with these findings. As demonstrated in the field cases of Farm 1 to Farm 4, if strict measures were taken, ASF can be contained in one area and systematically eliminated until the whole herd regained negative status. For example, in Farm 1, which was most heavily contaminated among four herds, 5 out 10 barns $(2,4,7,8,9)$ remained negative during the eradication process. This is also consistent with field observations that solid barriers like concrete walls can effectively block the transmission of ASFV between pens. This also supports the idea that ASF is not likely to be an airborne disease ${ }^{8}$. Environmental contamination of ASFV was relatively low, as was reported that introduction of negative pigs into contaminated pens 3, 5, 7 days after ASFV infected pigs were removed did not result in subsequent infection ${ }^{5}$. This seemed contradictory with results of Farm 2 but was consistent with results of Farm 1, 3 and 4. In the case of Farm 2, whole surface of environmental samples in Gestation Room 1 found qPCR positive almost every day until day 28 when a new disinfection method using sodium hypochlorite was implemented. We hypothesize that the positive qPCR results were due to non-degraded ASFV DNA but not infectious ASFV particles. Our study showed that sodium hypochlorite was more effective in breaking down DNA of ASFV making sodium hypochlorite more favorable to use by avoiding confusing positive DNA with infectious ASFV particles especially when evaluating the cleaning and disinfection outcome. This is also consistent with findings of a recent report that sodium hypochlorite and chlorine work well in damaging ASFV DNA ${ }^{1}$.

These characteristics of ASFV constitute the scientific foundation or our method and vice versa, our results supported these findings. Our method has been constantly upgraded including establishment of quick test labs and using sodium hypochlorite for disinfectants, etc, since the first case of ASFV detection. As showed by the TTNH and retention rate results, the latest removal in Farm 4 holds almost $99 \%$ of the herd. This can be attributed to several key points in the application and constant upgrading of the method. The key points are listed below.

1) Early detection is essential. Early detection means lower levels of contamination and less likelihood of spread of the virus. Compared with the cases of Farm 1 and Farm 2 in 2019, the qPCR results of first ASFV detection in Farm 3 and Farm 4 in 2020 were of higher CT values, which turned out to be smaller number of pigs infected (only 1 detected pig in Farm 3 and Farm 4 and retention rate was over 99\%.). This early detection contributed to a great extent to the successful implementation of the method.

Early detection can be attributed to several improvement in management. After 2019, each sow farm was equipped with qPCR machine and skilled personnel near the farm, which allows daily monitoring of clinically abnormal pigs, environment, personnel, and incoming supplies. In the cases of Farm 1 and Farm 2 in 2019, samples had to be sent in a qualified lab for analysis, which caused one day delay of 
results and higher chances of spread. NOR, OF, or PCAS samples were chosen for early detection and lymph node for confirmation. Zhao et al reported that viral DNA appeared 1 3 days earlier in oral fluid than in blood in pigs infected by Chinese strain via contact ${ }^{11}$. Also, NOR, OF, or PCAS samples are collected in a less invasive way and have less chances of contamination of the pigs and environment as compared to blood samples. As was shown in figure 1, average CT value for $L N$ samples was significantly lower than that of NOR or PCAS samples. Since qPCR results with a Ct value of $₫ 35$ was normally distributed, LN samples with lower CT values and expected higher concentrations of ASFV DNA were chosen as more reliable samples for ASFV DNA confirmation. Moreover, aggregation of samples made the method cost effective. As was shown in figure 1, aggregation of 2048 samples and of 16 samples if initial CT value is of $\sim 20$ CT values and of $\sim 33$ respectively would not change the qPCR results.

2) Precision evaluation of the herd. Upon detecting ASFV in the herd, precision evaluation of the degree of contamination must be carried out promptly. Four tools are useful in the evaluation: electronic maps, whole herd sampling and paired sampling \& testing and qPCR.

Electronic maps and whole herd sampling together generate an accurate picture of ASFV situation in the herd (figure s4). This helps make decisions in precision removal, tracing sources of contamination, and restarting production. For example, in Farm 3, whole herd sampling suggested that only Stall A87 was positive for one day (day 0), but interestingly, the internal surface of feeder outlet was found qPCR positive with CT value of 39.53 in day 13. Epidemiological investigation proved that feed plant was contaminated in day-20, so the source of infection might be attributed to the contaminated feed. This example highlights the importance of whole herd sampling, which is not easy to perform, in epidemiological tracing and decisions of resuming normal operations.

The concept of Paired sampling \& testing means once again sampling \& testing the same grid of the first round of Whole herd sampling, which is marked on the electronic map. At least two rounds of paired sampling \& testing were required to identify the ASFV infections. We observed in the field that some farms experienced repeated ASFV infection. Infected pigs via contact varied in time to manifest clinical signs $^{5}$, and some studies showed that virus shedding appeared earlier than clinical signs ${ }^{1}$, so it is important to conduct paired sampling and testing to avoid misdiagnosing assumed infected animals. As was seen in the case of Farm 2, an intermittent mode of ASFV detection was found probably due to not fully implemented paired sampling.

Similar method was used in the eradication of PRV. In the PRV eradication program, reestablishing QN breeding herd status after PRV infection requires repeated sampling and testing 30 days after the initial test $^{12}$. The underlying mechanism was similar, but the time interval between paired sampling \& testing in our practice stems precisely from the latent period of ASFV strain Georgia 2007, the prevalent strain in China ${ }^{13}$. The successful eradication of ASFV from herd proved the credibility of paired sampling \& testing. 
Although qPCR was widely used in the academic studies, it is uncommon to see the use of qPCR as a tool of swine disease diagnosis or monitoring in China. In less than two years of ASFV outbreak in China, our large production systems were equipped with qPCR machines and reagents. The qPCR test showed a superior advantage over traditional gel-based tests on aspects of sensitivity and promptness. Moreover, since $\mathrm{Ct}$ values are inversely proportional to the amount of target nucleic acid in the sample, qPCR results are indicative of level of viral shedding or contamination.

3) Precision removal was based on precision evaluation. After precision evaluation of level of contamination, individual pigs can be removed in a bio-secure manner that has the least chances of spreading the virus (figure s4). For example, the number of pigs removed were based on production type (Gestation, Farrowing and Wean to finish or GDU), number of pigs infected in one grid and Ct values. In the gestation, if Ct value was lower than 30 , and/ or more than two pigs were infected in the grid, the whole grid was depopulated. If CT value was $₫ 30$, the infected pig and two adjacent pigs were removed. This decision was made based on rough scientific estimation but gives staff clear operation instructions on site and make the method easy to implement, which is essential in eradication of ASFV from herd. Another example of precision removal based on precision evaluation is the design of pig removal routes. As is seen from figure s4, pig removal route was designed based on electronic maps and had least chances of spreading the virus.

In conclusion, we firstly developed an innovative, and systematic method, and successfully implemented it to eradicate ASFV in four farms with most herds retained (nearly $90 \%$ in recent two cases) for normal production. The successful eradication of ASFV in herds would greatly facilitate the control and eradication of ASFV in China and worldwide.

\section{Methods}

When first detection of ASFV occurs in the farm, whole herd sampling and qPCR tests were carried out to evaluate the disease status in the herd. Then infected pigs were accurately removed and environment decontaminated by the precision removal process. After one or more rounds of this method were applied until the whole herd remained negative for 7-14days.

\section{Farms}

Four different farms were chosen in the time order. First ASFV detection was in February $7^{\text {th }} 2019$, June $2^{\text {nd }} 2019$, July $2^{\text {nd }} 2019$, November $9^{\text {th }} 2019$ in Farm 1-4, respectively. For each farm, the study period last from the day of first ASFV infection detection to 7-14 days after the day of last positive qPCR result in the herd.

Farm 1 was a wean-finishing site holding 4231 growing pigs with average weight ranging from $7 \mathrm{~kg}$ to $130 \mathrm{~kg}$. 
Farm 2, a typical commercial sow farm, which had newly developed a herd by introducing 1484 gilts in the breeding gestation room. The breeding gestation room is part of a typical uniformed production line, which was designed to hold 3000 sows and include two breeding gestation rooms each with 1296 stalls and 10 farrowing rooms each with 60 crates. Infection was firstly detected in one breeding gestation room.

Farm 3 was a commercial farrow-wean sow farm with 5167 in production sows kept in two independent uniformed production lines as described above. Infection was firstly detected in one breeding gestation room.

Farm4 was also a commercial farrow-wean sow farm with 3928 in production sows kept in two independent uniformed production lines as described above. Infection was firstly detected in one breeding gestation room.

\section{Whole-herd sampling}

Detailed maps showing the precise layout of each farm were produced to inform the sampling protocol. Pigs and the environment were sampled and if one pig was diagnosed as ASFV positive, a whole herd (WH) sampling of pigs and environment was performed, meaning sampling each pig and surfaces in the building within 24 hours. Subsequent whole herd pig samples were collected seven to ten days later.

\section{Risk based early detection of infected pigs}

Two kinds of samples from pigs were collected with a modified method based on those used for PRRSV monitoring ${ }^{14}$. For early detection, all clinically abnormal pigs with signs including off-feed, fever, lethargy, hemorrhagic diarrhea, redness of skin, lameness, and abortion were sampled and tested. Swabs from nasal, oral, rectal (NOR), trough lips, and defecation area surface (pig contact area surface, PCAS) in the sow herd; or oral fluid (OF), trough lips, waterers, and defecation area surface and other floor surface (pig contact area surface, PCAS) swabs in the finishing pig herd were pooled together in a $2 \mathrm{ml}$ microtube as one sample. The pooled sample was only valid when at least three out of the five swab samples were successfully taken from the same pig. For confirmation of first ASFV DNA detection, especially when samples showed Ct values of higher than 35 , lymph node (LN) samples were collected using an innovative lymph node sample collector (Figure s1). In brief, the pig was held down and the needle-like collector was pierced into an inguinal lymph node. The sample was taken out by the barb of the collector and was then injected into a $2 \mathrm{ml}$ microtube as one lymph node sample.

\section{Whole-herd sampling of pigs}

The NOR samples from each sow in the farm were taken individually based on method described above after ASFV infection was confirmed. 


\section{Whole Surface (WS) sampling of supplies, personnel, and environment.}

Whole surface sampling can be literally defined as sampling the surface of supplies, personnel, and environment (Figure s2). A $20 \mathrm{~cm} \times 20 \mathrm{~cm}$ gauze soaked with $0.9 \%$ sodium chloride was used to wipe the surface of supplies, personnel and environment. For environmental sampling, a grid sampling frame was used. A grid represented 20 30 stalls in a gestation room, or a crate in a farrowing room, or a pen with solid walls in a wean-finishing site, or a functional room in the facility such as one dormitory or kitchen. Each pig and grid are clearly marked on the electronic map.

Ground surface, feeder, waterer, slats, and every object in the grid were wiped from top to the bottom. Each grid served as one sample. All samples from hair, face, nasal cavity, glasses, clothes, and boots of individual staff constituted a sample. For supplies, each category of incoming items was swabbed as one sample. The WS samples were put in a valve bag.

\section{Sample processing and aggregation of samples}

NOR or PCAS samples were diluted with $1 \mathrm{ml}$ of $0.9 \%$ sodium chloride and vortexed and then centrifuged at $4500 \mathrm{rpm}$ for 30 seconds. Supernatant was collected and stored at $-20^{\circ} \mathrm{C}$. The WS samples were squeezed for 30 seconds until the dilution were homogenized. The dilutant was then poured into a $1.5 \mathrm{ml}$ microtube and centrifuged at $4500 \mathrm{rpm}$ for 30 seconds. Supernatant was collected and stored at $-20^{\circ} \mathrm{C}$ for further use. lymph node samples were added with $500 \mathrm{ul}$ of $0.9 \%$ sodium chloride and homogenized with a homogenate machine. The homogenate was centrifuged at $4500 \mathrm{rpm}$ for 30 seconds and supernatant was collected and stored at $-20^{\circ} \mathrm{C}$ until further use.

No more than 5 NOR and PCAS or WS samples were aggregated as one. Each lymph node sample was tested individually.

\section{qPCR testing}

\section{DNA extraction}

DNA extraction was performed using a DNA extraction machine Gene Pure Pro 96 from Bioer company (Hangzhou, China) according to the manufacturer's instructions.

\section{qPCR testing}

$5 \mathrm{ul}$ of extracted DNA was added to 20ul of qPCR mix from MRD company (Beijing, China) or Thermo Fisher Scientific (Waltham, USA) and qPCR was performed in a Gentier 48E machine from Tianlong 
company (Xian, China) or Step one Plus from Thermo Fisher Scientific (Waltham, USA) according to the manufacturers' instructions. The procedure for qPCR test was as follows:

$50^{\circ} \mathrm{C}$ for 2 minutes, 1 cycle,

$95^{\circ} \mathrm{C}$ for 3 minutes, 1 cycle,

$95^{\circ} \mathrm{C}$ for 10 seconds, 45 cycles

$60^{\circ} \mathrm{C}$ for 20 seconds, 45 cycles

\section{Precision removal}

\section{Materials and equipment used for precision removal}

Facial masks, latex gloves, overalls, shoe covers, waterproof polyester clothing, vessels, and carts used for carrying dead pigs were purchased from local markets. Sodium hydroxide and sodium hypochlorite was purchased locally. Virkon was purchased from Lanxess (Cologne, Germany).

\section{Number of removed pigs}

The number of pigs removed was based on production type (gestation, farrowing, wean to finish or GDU), number of pigs infected in one grid, and Ct values of qPCR test.

In gestation, if the Ct value was lower than 30 , and/ or more than two pigs were infected in one grid, the whole grid was depopulated. If the Ct value was $₫ 30$, the infected pig and the two adjacent pigs were removed. In the farrowing room, regardless of $\mathrm{Ct}$ values, sows were removed by crate, and suckling piglets in the same crate were also removed. In the finishing site, if one pig was infected, the entire pen of pigs was removed. The two adjacent pens were only removed if the pens were not divided by solid walls.

\section{Precision Removal of the pigs}

Pigs were removed in a bio-secure manner. Sealed U-shape tunnel were made from waterproof polyester cloth (Figure s3) to move the pigs. The pigs were transferred using exclusive carts off the facility. After the pigs were removed, the supplies including gloves, overalls and cloth were incinerated. Afterwards, Virkon was applied in each grid.

\section{Paired sampling \& testing and subsequent daily monitoring and management}


Sampling and testing continued afterwards using the same grid as the first round of WH sampling as recorded on the electronic map. At least one round of subsequent sampling \& testing was required to eliminate the ASFV. More rounds were needed in the case of heavy contamination. Paired sampling and testing was ceased when previous round found no positive pigs.

After the herd (including pigs and the environment) remained negative for 21 consecutive days, clinically abnormal pigs with signs, such as being off-feed, fever, lethargy, hemorrhagic diarrhea, redness of skin, lameness, and abortion were tested daily. The NOR and PCAS samples were collected for ASFV qPCR test.

\section{Validation of maximum number of sample aggregation for valid nucleoid acid detection}

Verified LN, WS, NOR, and PCAS samples with initial Ct of $\sim 20$ and $\sim 33$ respectively were serially diluted 2 folds with PBS and quantified by qPCR for 2 replicates.

\section{Data collection}

Data including qPCR result and TTNH (time to negative herd, in which both pigs and environment were negative ) were collected from each farm. TTNH was determined by calculating the days from the first ASFV positive qPCR result until the last positive result.

\section{Declarations}

\section{Ethics approval and consent to participate}

All experimental protocols were approved by Ethical Committee of Swine Research Institute of New Hope Liuhe Co., Ltd. All methods were carried out in accordance with Nine Key Techniques for Prevention and Control of African Swine Fever and Resumption of Pig Production by National Pig Industry Technology System. All methods are reported in accordance with ARRIVE guidelines.

\section{Consent for publication}

Not applicable.

\section{Availability of data and materials}

The datasets used and/or analysed during the current study are available from the corresponding author on reasonable request.

\section{Competing interests}


The authors declare no competing interests.

\section{Funding}

This research was supported by Integration and Demonstration of Comprehensive Prevention and Control of ASFV by Ministry of Science and Technology (No. 2018YFC0840405), New Hope Group Science and Technology Leading 100-person Program Fund $\triangle \mathrm{NH} 201806)$, Shandong Provincial Key Laboratory Open Fund (SD2019BP102).

\section{Author contributions}

Zhichun Jason Yan created method and designed all the experiment. Xiaowen Li etc. carried out the action in Farm1 Farm 2, Farm 3, and Farm 4, respectively. Peng Li wrote the manuscript. All authors contributed to the production of the manuscript. All authors gave final approval for publication.

\section{Acknowledgement}

We thank Professor. John Deen from University of Minnesota and Professor Dirk U. PFEIFFER from City University of Hong Kong, Dr. TANG Hao from FAO, and Dr CUI Jixian for suggestions and proofreading.

\section{References}

1. Lang Gong, et al. African swine fever recovery in China, Vet Med Sci. 2020;00:1-4.

2. https://www.oie.int/en/animal-health-in-the-world/animal-diseases/african-swine-fever/

3. http://www.moa.gov.cn/nybgb/2017/dsq/201802/t20180201_6136188.htm

4. C. GUINAT et al. Experimental pig-to-pig transmission dynamics for African swine fever, virus, Georgia 2007/1 strain, Epidemiol Infect, 144(1): 25-34, 2016 Jan.

5. A S Olesen et al. short-time window for transmissibility of African swine fever virus from a contaminated environment, Transbound Emerg Dis, 2018 Aug;65(4):1024-1032. doi: 10.1111/tbed.12837.

6. C. GUINAT et al.Inferring within-herd transmission parameters for African swine fever virus using mortality data from outbreaks in the Russian Federation , Transbound Emerg Dis. 2018 Apr;65(2):e264-e271.doi: 10.1111/tbed.12748

7. H C de Carvalho Ferreira et al. Quantification of Airborne African Swine Fever Virus After Experimental Infection, Vet Microbiol,2013 Aug 30;165(3-4):243-51. doi: 10.1016/j.vetmic.2013.03.007.

8. Gallardo C, African swine fever (ASF) diagnosis, an essential tool in the epidemiological investigation, Virus Research 271 (2019) 197676.

9. C, Gallardo et al. Experimental infection of domestic pigs with African swine fever virus Lithuania 2014 genotype II field isolate, Transbound Emerg Dis, 2015 March 22, doi.org/10.1111/tbed.12346 
10. Claire Guinat, et al. Dynamics of African swine fever virus shedding and excretion in domestic pigs infected by intramuscular inoculation and contact transmission, Vet Research, September 2014, DOI: 10.1186/PREACCEPT-1922992841132370.

11. Dongming Zhao, etc. Replication and virulence in pigs of the first African swine fever isolated in China, Emerging Microbes \& Infections, January 2019, DOI: 10.1080/22221751.2019.1590128

12. Pseudorabies Eradication State-Federal-Industry Program Standards, USDA, Aphis

13. Jingyue Bao, etc., Genome comparison of African swine fever virus China/2018/Anhui XCGQ strain and related European p72 Genotype II strains, Transbound Emerg Dis 2019 May;66(3):1167-1176. doi: $10.1111 /$ tbed. 13124

14. Linhares et al., 2019 AVIA conference in Canada: Overview and applications of population-based monitoring protocols for PRRSv detection in breeding herds.

\section{Figures}

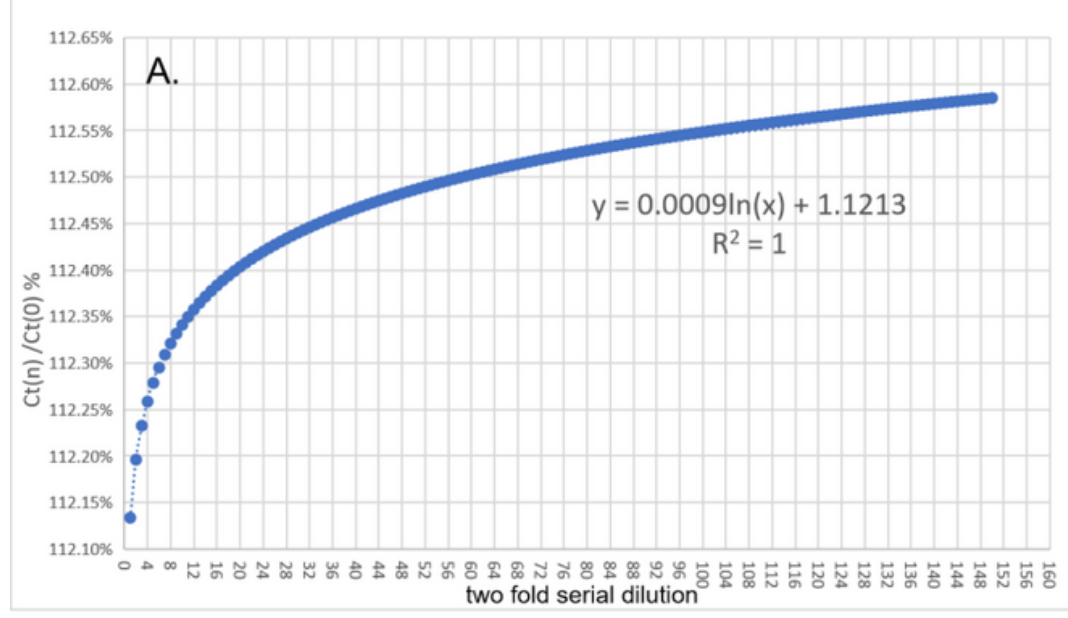

C.

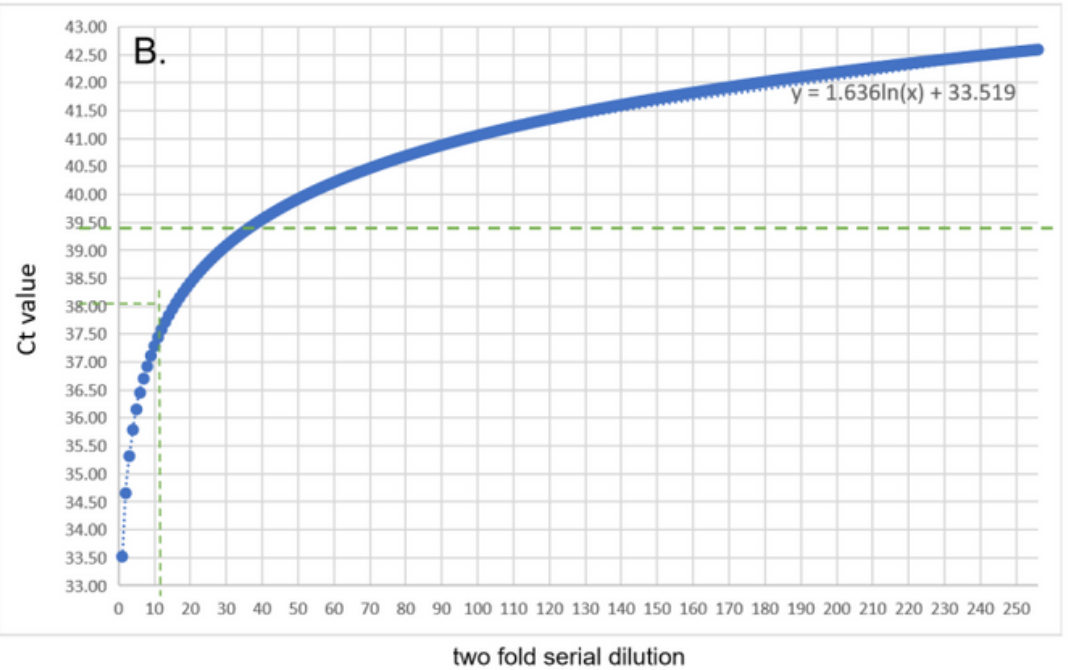

D.

two fold serial dilution

Figure 1 
Validation of maximum number of sample pooling for achieving acceptable sensitivity of ASFV DNA detection. Samples with original CT value of $\sim 20$ and $\sim 33$ respectively were serially diluted and measured by qPCR. Standard curve was established based on qPCR results (A) standard cure of samples with initial CT of $\sim 20$.(B) standard cure of samples with initial CT of $\sim 33$.Variations in CT values after serial dilution. (C) variation of samples with initial CT of $\sim 20$ (D) variation of samples with initial CT of $\sim 33$.

\begin{tabular}{|c|c|c|c|c|c|c|c|c|c|c|c|c|c|c|c|c|c|c|c|c|c|c|c|c|c|c|c|}
\hline \multicolumn{28}{|c|}{ TTNH of Farm 1} \\
\hline Date & $5 / 14$ & 5/15 & $5 / 16$ & 5/17 & 5/18 & $5 / 19$ & $5 / 20$ & $5 / 21$ & $5 / 22$ & $5 / 23$ & $5 / 24$ & $5 / 25$ & $5 / 26$ & $5 / 27$ & $5 / 28$ & $5 / 29$ & $5 / 30$ & $5 / 31$ & $6 / 1$ & $6 / 2$ & \begin{tabular}{l|l}
$6 / 3$ & 6 \\
\end{tabular} & \begin{tabular}{l|l}
$6 / 4$ & $6 / 1$ \\
\end{tabular} & \begin{tabular}{l|l}
$6 / 5$ & $6 / 6$
\end{tabular} & \begin{tabular}{|l|l|}
6 & $6 / 7$ \\
\end{tabular} & $6 / 8$ & $6 / 9$ & $6 / 10$ \\
\hline Room 1 & & & & & & & & & & & & & & & & & & & & & & & & & & & \\
\hline Room 2 (no pigs) & & & & & & & & & & & & & & & & & & & & & & & & & & & \\
\hline Room 3 & & & & & & & & & & & & & & & & & & & & & & & & & & & \\
\hline Room 5 & & & & & & & & & & & & & & & & & & & & & & & & & & & \\
\hline Room 6 & & & & & & & & & & & & & & & & & & & & & & & & & & & \\
\hline Room 7 & & & & & & & & & & & & & & & & & & & & & & & & & & & \\
\hline Room 8 & & & & & & & & & & & & & & & & & & & & & & & & & & & \\
\hline Room 9 & & & & & & & & & & & & & & & & & & & & & & & & & & & \\
\hline Supplies & & & & & & & & & & & & & & & & & & & & & & & & & & & \\
\hline Personnel & & & & & & & & & & & & & & & & & & & & & & & & & & & \\
\hline WS of external environment & & & & & & & & & & & & & & & & & & & & & & & & & & & \\
\hline
\end{tabular}

\begin{tabular}{|c|c|c|c|c|c|c|c|c|c|c|c|c|c|c|c|c|c|c|c|c|c|c|c|c|c|c|c|c|c|c|}
\hline B. & & & & & & & & & & & TTN & $\mathrm{HH}$ of & Far & $\mathrm{m} 2$ & & & & & & & & & & & & & & & & \\
\hline date & \begin{tabular}{|c|}
$7 / 1$ \\
0
\end{tabular} & \begin{tabular}{c|c}
$7 / 1$ & 7 \\
1 & \\
\end{tabular} & \begin{tabular}{c|c}
11 & $7 / 1$ \\
2 & 3 \\
\end{tabular} & \begin{tabular}{|c|}
$77 / 1$ \\
4
\end{tabular} & \begin{tabular}{c|c}
$7 / 1$ & $7 / 1$ \\
5 & 6 \\
\end{tabular} & $\begin{array}{c}7 / 1 \\
7\end{array}$ & \begin{tabular}{|c|c}
$7 / 1$ & $7 / 1$ \\
8 & 9 \\
\end{tabular} & \begin{tabular}{c|c|c|}
711 & 712 \\
9 & 0
\end{tabular} & \begin{tabular}{|c|c}
$7 / 2$ & 7 \\
1 & \\
\end{tabular} & \begin{tabular}{c|c}
$7 / 2$ & 712 \\
2 & 3 \\
\end{tabular} & \begin{tabular}{|c|c|c|}
$7 / 2$ & $7 / 2$ \\
4 & 5 \\
\end{tabular} & \begin{tabular}{|c|c|}
2 & 712 \\
6
\end{tabular} & \begin{tabular}{|c|c|c|}
$7 / 2$ & 77 \\
7 & 8
\end{tabular} & & \begin{tabular}{l|c}
$7 / 3$ \\
9 & $7 / 3$ \\
\end{tabular} & \begin{tabular}{l|c}
3 & $7 / 3$ \\
1
\end{tabular} & \begin{tabular}{|l|l|}
3 & 8 \\
1
\end{tabular} & \begin{tabular}{|l|l|}
$8 /$ & $8 /$ \\
2 & 3 \\
\end{tabular} & \begin{tabular}{|l|l|l|}
3 & $8 / 4$ \\
& 4 & \\
\end{tabular} & \begin{tabular}{|l|l|l|}
8 & $8 / 1$ \\
5 & 6 \\
\end{tabular} & \begin{tabular}{|l|l|l|l|l|}
8 & 8 \\
7 & 8 \\
\end{tabular} & $\mid$\begin{tabular}{l|l}
$\mid 8$ \\
9
\end{tabular} & \begin{tabular}{r|r}
$8 / 1$ & 8 \\
0 &
\end{tabular} & \begin{tabular}{|l|l|}
$8 / 1$ & $8 / 1$ \\
1 & 2 \\
\end{tabular} & \begin{tabular}{l|c|c|c|}
1 & $8 / 1$ \\
2 & 8 & 4
\end{tabular} & \begin{tabular}{|c|c|}
$8 / 1$ & $8 / 1$ \\
4 & 5 \\
\end{tabular} & \begin{tabular}{|c|c|c|}
$3 / 1$ & $8 / 1$ \\
5 & 6
\end{tabular} & \begin{tabular}{|l|l|}
$8 / 1$ & 8 \\
7 & 8
\end{tabular} & \begin{tabular}{r|r}
$8 / 1$ & $8 / 1$ \\
8 & 9 \\
\end{tabular} & \\
\hline Pigs in Gestation & & & & & & & & & & & & & & & & & & & & & & & & & & & & & & \\
\hline stalls in gestation & & & & & & & & & & & & & & & & & & & & & & & & & & & & & & \\
\hline Pigs in Gestation & & & & & & & & & & & & & & & & & & & & & & & & & & & & & & \\
\hline $\begin{array}{c}\text { stalls in gestation } \\
2\end{array}$ & & & & & & & & & & & & & & & & & & & & & & & & & & & & & & \\
\hline GDU & & & & & & & & & & & & & & & & & & & & & & & & & & & & & & \\
\hline $\begin{array}{l}\text { office building \& } \\
\text { facilities }\end{array}$ & & & & & & & & & & & & & & & & & & & & & & & & & & & & & & \\
\hline personnel & & & & & & & & & & & & & & & & & & & & & & & & & & & & & & \\
\hline $\begin{array}{l}\text { environment } \\
\text { outside Line } 1\end{array}$ & & & & & & & & & & & & & & & & & & & & & & & & & & & & & & \\
\hline C. & & & & & & & & & & & TTNH & $\mathrm{H}$ of & Far & & & & & & & & & & & & & & & & & \\
\hline $\mathrm{Dz}$ & & & & & $1 / 28$ & & 129 & $1 / 3 C$ & & $2 / 2$ & $2 / 5$ & & $2 / 10$ & & $2 / 11$ & & $/ 16$ & & $/ 17$ & $2 / 18$ & & $2 / 19$ & & $2 / 20$ & $2 / 2$ & 21 & $2 / 22$ & & $2 / 23$ & \\
\hline Line 1-Gest & ition & $1-$ & $A 87$ & & & & & & & & & & & & & & & & & & & & & & & & & & & \\
\hline Clinically ab & orm & nal & pigs & & & & & & & & & & & & & & & & & & & & & & & & & & & \\
\hline Threater & ed a & area & & & & & & & & & & & & & & & & & & & & & & & & & & & & \\
\hline WS except th & eate & nec & are & & & & & & & & & & & & & & & & & & & & & & & & & & & \\
\hline Sup & lies & & & & & & & & & & & & & & & & & & & & & & & & & & & & & \\
\hline Pers & nne & & & & & & & & & & & & & & & & & & & & & & & & & & & & & \\
\hline Feeder ol & et ir & $\mathrm{A} 8$ & & & & & & & & & & & & & & & & & & & & & & & & & & & & \\
\hline D. & & & & & & & & & & & TTNH & $\mathrm{H}$ of & Far & & & & & & & & & & & & & & & & & \\
\hline date & & $6 / 4$ & $6 / 5$ & $6 / 6$ & $6 / 7$ & $6 / 8$ & $6 / 12$ & $6 / 13$ & $6 / 14$ & $6 / 15$ & $|6 / 16| 6$ & $6 / 176$ & $6 / 18$ & $6 / 19$ & $96 / 2$ & $206 / 2$ & $21 \mid 6$ & $6 / 22$ & $6 / 23$ & $6 / 24$ & $6 /\left.25\right|^{6}$ & \begin{tabular}{|c|}
$6 / 2$ \\
6 \\
\end{tabular} & \begin{tabular}{c|}
$6 / 2$ \\
7 \\
\end{tabular} & \begin{tabular}{|c|}
$6 / 2$ \\
8 \\
\end{tabular} & \begin{tabular}{|c|c|}
$6 / 2$ & 6 \\
9 & 0 \\
\end{tabular} & \begin{tabular}{l|l}
$6 / 3$ & $7 /$ \\
0 & 7 \\
\end{tabular} & $/ 17$ & \begin{tabular}{l|l}
12 & 7. \\
\end{tabular} & $7 / 3 \mid 7$ & $1 / 4$ \\
\hline Stall H26 & & & & & & & & & & & & & & & & & & & & & & & & & & & & & & \\
\hline $\begin{array}{c}\text { Pigs in threater } \\
\text { area }\end{array}$ & & & & & & & & & & & & & & & & & & & & & & & & & & & & & & \\
\hline $\begin{array}{c}\text { Clinically abnor } \\
\text { pigs }\end{array}$ & & & & & & & & & & & & & & & & & & & & & & & & & & & & & & \\
\hline WS of environm & & & & & & & & & & & & & & & & & & & & & & & & & & & & & & \\
\hline Supplies & & & & & & & & & & & & & & & & & & & & & & & & & & & & & & \\
\hline Personnel & & & & & & & & & & & & & & & & & & & & & & & & & & & & & & \\
\hline
\end{tabular}


Figure 2

TTNH (time to negative herds) in Farm 1(A), 2(B), 3(C), 4(D) respectively. Red box meant positive qPCR result and green box represented negative result. White box meant not detected.

\section{Retention Rate}

\section{$120.00 \%$}

$100.00 \%$

$\mathbf{8 0 . 0 0 \%}$

\begin{tabular}{|l|l|}
\hline $\mathbf{6 0 . 0 0 \%}$ & $69.70 \%$ \\
$\mathbf{4 0 . 0 0 \%}$ & \\
\hline $\mathbf{2 0 . 0 0 \%}$ & \\
\hline $\mathbf{0 . 0 0 \%}$ & \\
\hline
\end{tabular}

Farm1

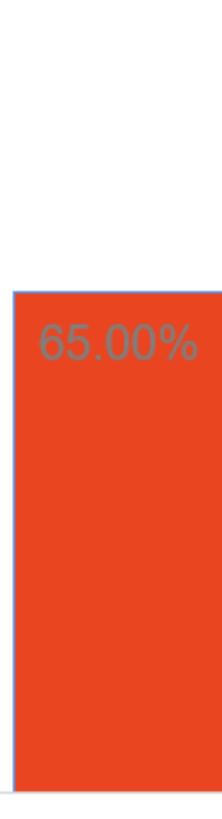

Farm2

\section{$99.40 \%$}

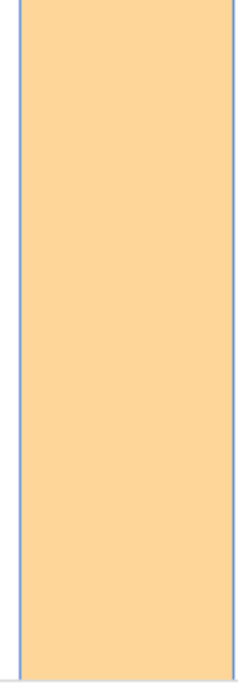

Farm3

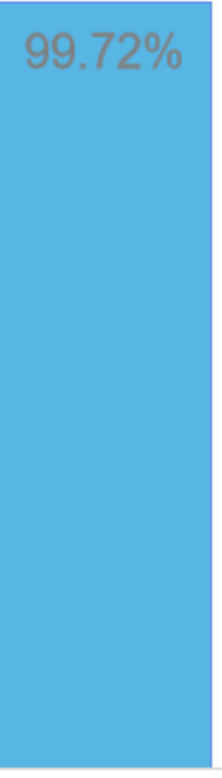

Farm4

Figure 3

Retention rate (=number of retained sows/ number of sows prior to detection) in $\mathbf{4}$ herds. 